\title{
Clinical Study \\ Electrocardiogram Changes of Donepezil Administration in Elderly Patients with Ischemic Heart Disease
}

\author{
Deguo Wang $\mathbb{D}^{1}{ }^{1}$ Yong Wu, ${ }^{1}$ Ancai Wang, ${ }^{1}$ Yueyun Chen, ${ }^{1}$ Ting Zhang, ${ }^{2}$ and Nengwei Hu ${ }^{3}$ \\ ${ }^{1}$ Department of Gerontology, Yijishan Hospital of Wannan Medical College, Wuhu, Anhui 241001, China \\ ${ }^{2}$ Department of Psychology, Wannan Medical College, Wuhu, Anhui 241001, China \\ ${ }^{3}$ Department of Pharmacology and Therapeutics, Institute of Neuroscience, Trinity College Dublin, Dublin, Ireland
}

Correspondence should be addressed to Deguo Wang; wangdeguo2005@hotmail.com

Received 6 January 2018; Accepted 25 February 2018; Published 23 April 2018

Academic Editor: Julian Bostock

Copyright (C) 2018 Deguo Wang et al. This is an open access article distributed under the Creative Commons Attribution License, which permits unrestricted use, distribution, and reproduction in any medium, provided the original work is properly cited.

Objective. Donepezil, a widely used cholinesterase inhibitor for treating Alzheimer's disease, has been reported to induce bradyarrhythmias and torsade de pointes. In this study, we aimed at determining electrocardiogram changes of donepezil administration in elderly patients with ischemic heart disease, who tend to suffer from cognitive disorders. Methods. Sixty patients with ischemic heart disease and mild cognitive impairment were treated with donepezil $(5 \mathrm{mg} /$ day) and followed up for at least four weeks. A twenty-four-hour ambulatory electrocardiogram was performed for the analysis of heart rate variability. The ECG parameters including heart rate (HR), PR and RR intervals, QT interval, and QRS duration were recorded at the baseline and after donepezil administration. Results. Donepezil administration resulted in significant reduction in mean HR and the lowest HR and prolongation of PR and RR intervals, whereas it had no significant effects on QRS duration and QT parameters including QT, corrected QT interval, QT dispersion, and $\mathrm{T}_{\text {peak-end }}$ interval. HRV analysis showed that donepezil administration significantly improved parasympathetic function, indicated by decreased low/high frequency (LF/HF) ratio and high frequency (HF) components and oscillation of RR intervals. Conclusions. These data demonstrated that donepezil administration decreased HR, prolonged PR interval, and increased parasympathetic function without affecting QRS duration and QT intervals, suggesting that it can be used safely in elderly patients with ischemic heart disease.

\section{Introduction}

Cardiovascular disease accounts for one-third of the deaths worldwide, particularly in aging population [1]. Some of them suffer from cognitive impairment. Therefore, combination therapy with cardiovascular and psychiatric drugs is very common [2]. Cholinesterase inhibitors are currently prescribed as the first line therapy for Alzheimer's disease (AD) and cognitive impairment [3, 4]. By inhibiting the enzyme acetylcholinesterase, cholinesterase inhibitors reduce breakdown of acetylcholine, which is associated with the improvement in memory function. As these drugs are continuously used, their side effects should be treated. Until now, the most of unwanted effects are involved mainly in the gastrointestinal system, which can be well tolerated $[3,5]$.
Theoretically, cholinesterase inhibitors can cause some cardiovascular effects, including bradycardia, conductive block, and QT prolongation [6,7], because the heart is rich in cholinesterase. Several case reports showed that donepezil could lead rarely to serious bradycardia needing pacemaker implantation and fatal ventricular arrhythmia (torsade de pointes $(\mathrm{TdP}))[7,8]$. However, the vagotonic effect of cholinesterase inhibitors might also protect the heart against ischemia and dysarrhythmias. Experimental studies suggested that donepezil improved the long-term survival of congestive heart failure rats by preventing pump failure and cardiac remodeling and attenuating atherogenesis $[9,10]$. Interestingly, a population-based study showed that donepezil actually significantly reduced risk of cardiac pacemaker insertion [11]. Another nationwide cohort study revealed that donepezil reduced 
the risk of myocardial infarction and death in subjects with $\mathrm{AD}$ [12]. In the present study, we examined the effects of donepezil on ECG parameters including heart rate (HR), QT interval, corrected QT interval (QTc), QT interval dispersion (QTd), and $\mathrm{T}_{\text {peak-end }}$ interval, which are associated with the risk of bradycardia and TdP.

Higher sympathetic and lower parasympathetic functions were found to be associated with lower cognitive performance in patients with $\mathrm{AD}$ [13] and all-cause mortality and arrhythmic death in patients with ischemic heart disease [14]. In the present study, we examined cardiac autonomic function using heart rate variability (HRV) analysis. HRV is the beat-to-beat oscillation of RR intervals in an electrocardiogram. Until now, it is a reliable tool for evaluating autonomic nervous system activities by characterizing the relative and absolute parasympathetic and sympathetic modulations and the sympathovagal balance of the heart [15].

In the present study, we focused on ischemic heart disease patients complicated with mild cognitive impairment (MCI). By analyzing HRV and electrocardiogram parameters, we determined whether donepezil is associated with an increased risk of bradycardia and ventricular arrhythmia.

\section{Methods}

2.1. Study Subjects. The study included elderly patients $(>65$ years) with established coronary artery disease with mild cognitive impairment, who visited our hospital between January 1, 2015, to September 31, 2017. Patients would be selected to the study if they had not been described with any cholinesterase inhibitors within 3 months according to the previous report [7]. Ischemic heart disease was diagnosed, including pervious myocardial infarction, stable angina pectoris, abnormal cardiac exercise test, or plaque by prior imaging in at least one coronary artery. The abnormal cardiac exercise test results including horizontal or down sloping ST depression, J-point elevation, angina pectoris, and blood pressure decline confirm the presence of ischemic heart disease [16].

Mild cognitive impairment (MCI) was diagnosed by a psychiatrist according to Chinese guidelines for diagnosis and management of cognitive impairment and dementia [17]. Mini-Mental State Examination (MMSE) [18] and Alzheimer's Disease Cooperative Study Scale for activities of daily life in MCI (ADCS-MCI-ADL) were used as a brief screening tool for MCI. The exclusion criteria were patients with atrial fibrillation, acute myocardial infarction, or acute coronary syndrome in the past 3 months; patients with bradycardia; and patients with artificial cardiac pacing or using antiarrhythmic agents.

2.2. Study Procedures. Before participation, all patients gave written informed consent to participate in the study, which was approved by the Institutional Research Ethics Committee, in accordance with Chinese guidelines for ethical review of drug clinical trials. After careful history taking, examination and laboratory evaluation (plasma glucose, potassium, magnesium and calcium), electrocardiogram (ECG), and Holter ECG measurement, the selected patients began to take donepezil once a day ( $5 \mathrm{mg}$ ) continuously for at least four weeks. During the 4-week follow-up, if the patients complained of syncope, palpitation, fatigue, or weakness, they were recalled to hospital and ECG measurement was taken immediately. Because QTc tends to respond to some drugs and electrolytes [15], the readmission was postponed if subjects had to take these drugs additionally or had electrolyte disturbances. Once the follow-up was completed in the subjects, ECG and Holter ECG was measured immediately.

2.3. Electrocardiogram Measurement. All patients were recorded a 12-lead surface ECG measurement by using $25 \mathrm{~mm} /$ second paper speed and standardized at $0.1 \mathrm{mV} / \mathrm{mm}$ after the patients had rested for at least 10 minutes in the supine position. ECG parameters, including $\mathrm{HR}, \mathrm{PR}, \mathrm{QT}$, QTc, QTd, and T peak-to-end ( $\mathrm{T}_{\text {peak-end }}$ ) interval, and QRS duration were calculated by two examiners blinded to the clinical characteristics of patients and groups [19]. The QT interval was corrected for the heart rate by using Bazett's formula (QTc $=\mathrm{QT} /$ square root of RR). QTd is defined as the difference between the maximum and minimum QT interval measured on the 12-lead. $\mathrm{T}_{\text {peak-end }}$ interval was measured in lead V5 from the peak of the T wave to the end of the T wave, defined as the point of return to the T-P baseline. If $U$ waves were present, we defined the end of the T wave as the nadir of the curve between the $\mathrm{T}$ and $\mathrm{U}$ waves.

\subsection{Holter ECG and HRV Measurement. A 12-leads Holter} electrocardiogram (DMS300-4, Diagnostic Monitoring Software, NV, USA) was performed in the participants for 24 hours. Because bradycardia is a relatively common occurrence in older patients after using cholinesterase inhibitors $[6,7]$, Holter ECG parameters such as mean heart rate (HR), the lowest HR, and the longest RR were determined.

HRV measurement was carried out before and four weeks after donepezil administration. The electrocardiogram data were analyzed off-line for HRV using DMS (Diagnostic Monitoring Software, USA). The resultant tachogram underwent 512 point fast Fourier transform with a Hanning window to calculate the power spectrum of HRV, with the low frequency (LF) component at $0.040-0.150 \mathrm{~Hz}$ and the high frequency (HF) component at $0.150-0.401 \mathrm{~Hz}$. A typical spectrum of RR series is characterized by two main components: the LF component and the HF component. The HF component reflects cardiac vagal tone, whereas the LF component reflects both sympathetic and parasympathetic modulation of the heart rate. The $\mathrm{LF} / \mathrm{HF}$ ratio is an index of sympathetic activity [15].

2.5. Statistical Analysis. Data were presented as the number (n) for nominal variables and as the mean \pm SD for continuous variables. The distribution characteristics of the variables were evaluated by the Kolmogorov-Smirnov test. 
TABLE 1: Demographics and baseline characteristics of patients.

Age (year)

Gender (male/female)

$75.7 \pm 5.8$

$37 / 23$

Follow-up period (days)

$\mathrm{SBP}(\mathrm{mm} \cdot \mathrm{Hg})$

DBP $(\mathrm{mm} \cdot \mathrm{Hg})$

MMSE

ADCS-MCI-ADL

HR (beats/min)

LVEF (\%)

Glucose $(\mathrm{mmol} / \mathrm{L})$

Potassium $(\mathrm{mmol} / \mathrm{L})$

Magnesium (mmol/L)

Calcium $(\mathrm{mmol} / \mathrm{L})$

Note. Values are shown as mean \pm SD. SBP: systolic blood pressure; DBP: diastolic blood pressure; MMSE: Mini-Mental State Examination; ADCSMCI-ADL: Alzheimer's Disease Cooperative Study Scale for activities of daily living in MCI; LVEF: left ventricular ejection fraction.

TABLe 2: Holter electrocardiogram and HRV at baseline and 4 weeks after donepezil intervention.

\begin{tabular}{lcccc}
\hline & Baseline & Donepezil & $t$ & $p$ \\
\hline Mean HR (beats/min) & $74.5 \pm$ & $68.1 \pm$ & 3.2260 & 0.0013 \\
The lowest HR & 11.3 & 10.4 & & \\
(beats/min) & $53.2 \pm 6.4$ & $50.3 \pm 6.8$ & 2.4056 & 0.0177 \\
Longest RR interval(s) & $1.36 \pm$ & $1.47 \pm$ & & \\
LF $\left(\mathrm{ms}^{2}\right)$ & 0.77 & 0.84 & 0.7477 & 0.4561 \\
HF $\left(\mathrm{ms}^{2}\right)$ & $8.7 \pm 8.5$ & $8.9 \pm 7.7$ & 0.1351 & 0.8928 \\
LF/HF ratio & $4.6 \pm 5.3$ & $6.8 \pm 4.6$ & 2.4283 & 0.0152 \\
& $2.14 \pm$ & $1.66 \pm$ & 2.3897 & 0.0169 \\
\hline
\end{tabular}

Note. Values are shown as mean $\pm \mathrm{SD}$. HR, heart rate; LF, low frequency; $\mathrm{HF}$, high frequency.

If the variables were of normal distribution, comparisons were tested by Student's $t$-test. Otherwise, comparisons were tested for statistical significance by using the MannWhitney $U$ test for nonnormal distribution data. Statistical analyses were performed using the SPSS software, version 16.0. The differences were considered to be significant at $P<0.05$.

\section{Results}

A total of 60 elderly patients were enrolled and completed the study including 37 male and 23 female subjects. The demographics and baseline characteristics of the patients are shown in Table 1. Blood pressure and heart rate of all individuals prior to donepezil were normal. The values of plasma glucose and plasma ions such as potassium, magnesium, and calcium were within normal range. The average follow-up period was $35.1 \pm 4.6$ days. During the period of follow-up, no any donepezil-related adverse effects were reported such as syncope, palpitation, fatigue, and weakness. Moreover, there were no bradycardia events leading to rehospitalization.

As shown in Table 2, mean HR and the lowest HR significantly reduced after donepezil administration compared
TABLE 3: ECG parameters at baseline and 4 weeks after donepezil intervention.

\begin{tabular}{lcccc}
\hline & Baseline & Donepezil & $t$ & $p$ \\
\hline HR (beats/min) & $76.8 \pm 10.8$ & $72.1 \pm 12.7^{*}$ & 2.1838 & 0.0310 \\
PR interval (ms) & $172.3 \pm 21.3$ & $182.6 \pm 25.8^{*}$ & 2.3847 & 0.0187 \\
QRS duration (ms) & $98.2 \pm 15.3$ & $96.6 \pm 24.3$ & 0.5918 & 0.5551 \\
QT interval (ms) & $378.3 \pm 31.3$ & $383.8 \pm 32.4$ & 0.9457 & 0.3462 \\
QTc (ms) & $415.1 \pm 35.7$ & $424.3 \pm 37.2$ & 1.0858 & 0.7529 \\
QTd (ms) & $64.5 \pm 10.7$ & $67.2 \pm 11.3$ & 1.4082 & 0.1591 \\
$\mathrm{~T}_{\text {peak-end }}(\mathrm{ms})$ & $75.3 \pm 12.7$ & $77.3 \pm 14.3$ & 0.8100 & 0.4179 \\
\hline
\end{tabular}

Note. Values are shown as mean \pm SD. HR, heart rate; QRS, QRS complexes; QTc, corrected QT intervals; QTd, QT dispersion; $\mathrm{T}_{\text {peak-end, }}$ T peak-to-end.

with baseline $(P<0.05)$. The longest $\mathrm{RR}$ interval was significantly prolonged with respect to baseline $(P<0.05)$. There was no significant difference in LF with or without donepezil administration. HF increased significantly after donepezil intervention. As a result, the LF/HF ratio decreased significantly after donepezil administration.

As shown in Table 3, the mean heart rate significantly reduced after donepezil intervention $(P<0.05)$. The mean PR interval was significantly prolonged after donepezil administration $(P<0.05)$. Moreover, the QRS duration and QT parameters including QT, QTc, QTd, and $\mathrm{T}_{\text {peak-end }}$ interval did not change significantly as a result of donepezil treatment and discontinuation.

\section{Discussions}

In the present study, we observed the cardiac effects of donepezil administration in patients with ischemic heart disease and focused on the risk of bradycardia and ventricular arrhythmia. Our data revealed two findings: (1) donepezil increased cardiac vagal tone and reduced cardiac sympathetic activity; (2) donepezil prolonged RR and PR interval without changing QRS duration and QT intervals.

Imbalance of the autonomic nervous system with higher sympathetic and lower parasympathetic function occurs in both $\mathrm{AD}$ and ischemic heart disease $[13,14]$. Treatment with acetylcholinesterase inhibitors may lead to bradycardia by potentiating cardiac vagal activity [20]. Previous studies suggested that treatment for $\mathrm{AD}$ with acetylcholinesterase inhibitors might result in bradyarrhythmias even needing pacemaker implantation $[6,7,21]$. However, these studies defined bradycardia as heart rate lower than 60 beats/minute. Most subjects had no symptomatic response to the slow heart rate. Among them, in only a few serious cases, artificial pacemakers had been implanted $[7,11,21]$. In the present study, we founded that donepezil decreased mean HR and the lowest HR and prolonged the longest RR interval. However, we did not observe serious bradycardia needing donepezil retraction or pacemaker implantation.

Because overexcited sympathetic function plays an important role in ischemic heart disease, the vagotonic stimulation of administrated acetylcholinesterase inhibitors might also provide beneficial effects [22]. Actually, recent studies provided strong evidences that donepezil reduces the risk of myocardial infarction and pacemaker implantation in 
subjects with $\mathrm{AD}[11,11,12]$. In two retrospective cohort studies on dementia patients which involved in East Asian, acetylcholinesterase inhibitors are associated with a decreased risk of an acute coronary syndrome event and ischemic stroke $[23,24]$. These cardiac benefits of acetylcholinesterase inhibitors might be associated with autonomic modulation against heart diseases. In the present study, our data showed that donepezil administration significantly increased HF and decreased LF/HF ratio, suggesting a shift in autonomic modulation from sympathetic to vagal tone.

Torsade de pointes (TdP) is a fatal ventricular arrhythmia and is associated with prolongation of QT interval which reflects enhanced repolarization dispersion in the myocardium [25]. QT prolongations are thought to be a marker of the fatal ventricular arrhythmia and related to multiple factors, such as aging, hypopotassium, taking antiarrhythmic drugs, and ischemic heart disease $[26,27] . \mathrm{T}_{\text {peak-end }}$ has been proposed to transmural dispersion of repolarization, which is related to the vulnerability of ventricular arrhythmias [28]. Prolongation of $\mathrm{T}_{\text {peak-end }}$ interval has been shown to be associated with ventricular arrhythmias in various cardiac disorders including long QT syndrome [29] and ischemic heart disease [30]. It is documented in a few case reports that donepezil prolonged QT interval and caused TdP $[8,27]$. These results could not reflect the pervasive impact of donepezil intervention because some cases had specific disease backgrounds such as unknown sinus sick syndrome, paroxysmal atrial fibrillation, myocardial infarction, or combination therapy with negative chronotropic drugs $[27,31]$. On the contrary, Isik and Igeta $[32,33]$ reported that donepezil administration did not change QT and QTc interval in patients with AD compared with baseline. In the present study, no significant difference in QT, QTc, QTd, and $\mathrm{T}_{\text {peak-end }}$ interval was detected after 4 weeks of donepezil intervention. These findings suggested that donepezil had no such remarkable effect on ventricular repolarization even in patients with stable coronary artery disease as documented in previous case reports.

\section{Limitations}

Several limitations of the present study should be stated. First, it is a small sample longitudinal study which included only a small number of subjects. Second, we could not deduce the long-term effects of electrocardiogram and HRV because we observed only the effects of donepezil administration for about one month. During such a short period, no any donepezil-related side effects such as bradycardia, conductive block, and QT prolongation had been reported as previously documented $[7,34]$. Third, the selected subjects in this study were mainly patients with ischemic heart disease and mild cognitive impairment. Some of them may have other complicated diseases such as hypertension, diabetes mellitus, and cerebral vascular disease. Therefore, it is difficult to analyze electrocardiogram changes in different complicated diseases. Further prospective studies are needed in the future including more subjects and longer time to determine the electrocardiogram changes of donepezil administration.

\section{Conclusions}

Although there are several limitations in this study, it is a novel study focused on patients with ischemic heart disease to determine the effects of donepezil intervention on the risk of bradyarrhythmias and ventricular arrhythmia. This study demonstrated that donepezil causes mild decrease in heart rate and prolongation of PR interval without affecting QRS duration and QT intervals, suggesting that it can be used safely in elderly patients with a history of ischemic heart disease.

\section{Conflicts of Interest}

The authors declare that they have no conflicts of interest.

\section{Acknowledgments}

This work was supported by grants from the National Natural Science Foundation of China (81670301 and 81471114).

\section{References}

[1] GBD Mortality and Causes of Death Collaborators, "Global, regional, and national age-sex specific all-cause and causespecific mortality for 240 causes of death, 1990-2013: a systematic analysis for the global burden of disease study 2013," The Lancet, vol. 385, no. 9963, pp. 117-171, 2015.

[2] Global Burden of Disease Study Collaborators, "Global, regional, and national incidence, prevalence, and years lived with disability for 301 acute and chronic diseases and injuries in 188 countries, 1990-2013: a systematic analysis for the global burden of disease study 2013," The Lancet, vol. 386, no. 9995, 2015.

[3] R. A. Hansen, G. Gartlehner, A. P. Webb, L. C. Morgan, C. G. Moore, and D. E. Jonas, "Efficacy and safety of donepezil, galantamine, and rivastigmine for the treatment of alzheimer's disease: a systematic review and meta-analysis," Clinical Interventions in Aging, vol. 3, no. 2, pp. 211-225, 2008.

[4] A. C. Tricco, C. Soobiah, S. Berliner et al., "Efficacy and safety of cognitive enhancers for patients with mild cognitive impairment: a systematic review and meta-analysis," Canadian Medical Association Journal, vol. 185, no. 16, pp. 1393-1401, 2013.

[5] K. R. McCain, T. S. Sawyer, and H. A. Spiller, "Evaluation of centrally acting cholinesterase inhibitor exposures in adults," Annals of pharmacotherapy, vol. 41, no. 10, pp. 1632-1637, 2007.

[6] D. M. Malone and J. Lindesay, "Cholinesterase inhibitors and cardiovascular disease: a survey of old age psychiatrists' practice," Age and Ageing, vol. 36, no. 3, pp. 331-333, 2007.

[7] L. Y. Park-Wyllie, M. M. Mamdani, P. Li, S. S. Gill, A. Laupacis, and D. N. Juurlink, "Cholinesterase inhibitors and hospitalization for bradycardia: a population-based study," PLoS Medicine, vol. 6, no. 9, article e1000157, 2009.

[8] A. Tanaka, S. Koga, and Y. Hiramatsu, "Donepezil-induced adverse side effects of cardiac rhythm: 2 cases report of atrioventricular block and torsade de pointes," Internal Medicine, vol. 48, no. 14, pp. 1219-1223, 2009.

[9] M. Li, C. Zheng, T. Kawada et al., "Donepezil markedly improves long-term survival in rats with chronic heart failure 
after extensive myocardial infarction," Circulation Journal, vol. 77, no. 10, pp. 2519-2525, 2013.

[10] K. Inanaga, T. Ichiki, R. Miyazaki et al., "Acetylcholinesterase inhibitors attenuate atherogenesis in apolipoprotein e-knockout mice," Atherosclerosis, vol. 213, no. 1, pp. 52-58, 2010.

[11] A. R. Huang, C. J. Redpath, and C. van Walraven, "The influence of cholinesterase inhibitor therapy for dementia on risk of cardiac pacemaker insertion: a retrospective, population-based, health administrative databases study in ontario, canada," BMC Neurology, vol. 15, no. 1, p. 66, 2015.

[12] P. Nordstrom, D. Religa, A. Wimo, B. Winblad, and M. Eriksdotter, "The use of cholinesterase inhibitors and the risk of myocardial infarction and death: a nationwide cohort study in subjects with alzheimer's disease," European Heart Journal, vol. 34, no. 33, pp. 2585-2591, 2013.

[13] Z. Nonogaki, H. Umegaki, T. Makino, Y. Suzuki, and M. Kuzuya, "Relationship between cardiac autonomic function and cognitive function in alzheimer's disease," Geriatrics \& Gerontology International, vol. 17, no. 1, pp. 92-98, 2017.

[14] J. T. Bigger Jr., J. L. Fleiss, L. M. Rolnitzky, and R. C. Steinman, "Frequency domain measures of heart period variability to assess risk late after myocardial infarction," American Journal of Cardiology, vol. 21, no. 3, pp. 729-736, 1993.

[15] E. Vandael, B. Vandenberk, J. Vandenberghe, R. Willems, and V. Foulon, "Risk factors for qtc-prolongation: systematic review of the evidence," International Journal of Clinical Pharmacy, vol. 39, no. 1, pp. 16-25, 2017.

[16] G. F. Fletcher, P. A. Ades, P. Kligfield et al., "Exercise standards for testing and training: a scientific statement from the American Heart Association," Circulation, vol. 128, no. 8, pp. 873-934, 2013.

[17] J. P. Jia, Y. H. Wang, and Z. X. Zhang, "Chinese guidelines for diagnosis and management of cognitive impairment and dementia (iii): psychometric selection," Zhonghua Yi Xue Za Zhi, vol. 91, pp. 735-741, 2011.

[18] Z. S. Nasreddine, N. A. Phillips, V. Bedirian et al., "The montreal cognitive assessment, moca: a brief screening tool for mild cognitive impairment," Journal of the American Geriatrics Society, vol. 53, no. 4, pp. 695-699, 2005.

[19] J. S. Perkiomaki, M. J. Koistinen, S. Yli-Mayry, and H. V. Huikuri, "Dispersion of QT interval in patients with and without susceptibility to ventricular tachyarrhythmias after previous myocardial infarction," Journal of the American College of Cardiology, vol. 26, no. 1, pp. 174-179, 1995.

[20] L. G. Howes, "Cardiovascular effects of drugs used to treat Alzheimer's disease," Drug Safety, vol. 37, no. 6, pp. 391-395, 2014.

[21] R. K. Hernandez, W. Farwell, M. D. Cantor, and E. V. Lawler, "Cholinesterase inhibitors and incidence of bradycardia in patients with dementia in the veterans affairs New England healthcare system," Journal of the American Geriatrics Society, vol. 57, no. 11, pp. 1997-2003, 2009.

[22] V. Kaushik, S. T. Smith, E. Mikobi, and M. A. Raji, “Acetylcholinesterase inhibitors: beneficial effects on comorbidities in patients with Alzheimer's disease," American Journal of Alzheimer's Disease and Other Dementias, vol. 33, no. 2, pp. 73-85, 2017.

[23] P. H. Wu, Y. T. Lin, P. C. Hsu, Y. H. Yang, T. H. Lin, and C. T. Huang, "Impact of acetylcholinesterase inhibitors on the occurrence of acute coronary syndrome in patients with dementia," Scientific Reports, vol. 5, no. 1, p. 15451, 2015.

[24] Y. T. Lin, P. H. Wu, C. S. Chen, Y. H. Yang, and Y. H. Yang, "Association between acetylcholinesterase inhibitors and risk of stroke in patients with dementia," Scientific Reports, vol. 6, no. 1, p. 29266, 2016.
[25] C. Antzelevitch, "Role of transmural dispersion of repolarization in the genesis of drug-induced torsades de pointes," Heart Rhythm, vol. 2, no. 11, pp. S9-S15, 2005.

[26] D. M. Roden, "Drug-induced prolongation of the qt interval," New England Journal of Medicine, vol. 350, no. 10, pp. 1013-1022, 2004.

[27] T. Takaya, M. Okamoto, K. Yodoi et al., "Torsades de pointes with qt prolongation related to donepezil use," Journal of Cardiology, vol. 54, no. 3, pp. 507-511, 2009.

[28] C. Antzelevitch, "Tpeak-tend interval as an index of transmural dispersion of repolarization," European Journal of Clinical Investigation, vol. 31, no. 7, pp. 555-557, 2001.

[29] M. Yamaguchi, M. Shimizu, H. Ino et al., "T wave peak-to-end interval and qt dispersion in acquired long qt syndrome: a new index for arrhythmogenicity," Clinical Science, vol. 105, no. 6, pp. 671-676, 2003.

[30] F. L. Burton and S. M. Cobbe, "Dispersion of ventricular repolarization and refractory period," Cardiovascular Research, vol. 50, no. 1, pp. 10-23, 2001.

[31] P. Bordier, S. Garrigue, S. S. Barold, N. Bressolles, S. Lanusse, and J. Clementy, "Significance of syncope in patients with Alzheimer's disease treated with cholinesterase inhibitors," Europace: European Pacing, Arrhythmias, and Cardiac Electrophysiology: Journal of the Working Groups on Cardiac Pacing, Arrhythmias, and Cardiac Cellular Electrophysiology of the European Society of Cardiology, vol. 5, no. 4, pp. 429-431, 2003.

[32] A. T. Isik, G. B. Yildiz, E. Bozoglu, A. Yay, and E. Aydemir, "Cardiac safety of donepezil in elderly patients with Alzheimer disease," Internal Medicine, vol. 51, no. 6, pp. 575-578, 2012.

[33] H. Igeta, Y. Suzuki, M. Tajiri, and T. Someya, "Cardiovascular pharmacodynamics of donepezil hydrochloride on the PR and QT intervals in patients with dementia," Human Psychopharmacology, vol. 29, no. 3, pp. 292-294, 2014.

[34] S. S. Gill, G. M. Anderson, H. D. Fischer et al., "Syncope and its consequences in patients with dementia receiving cholinesterase inhibitors: a population-based cohort study," Archives of Internal Medicine, vol. 169, no. 9, pp. 867-873, 2009. 


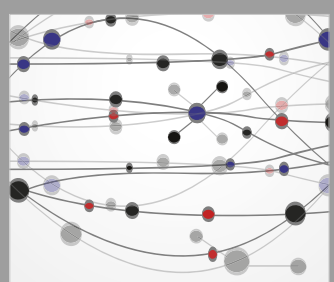

The Scientific World Journal
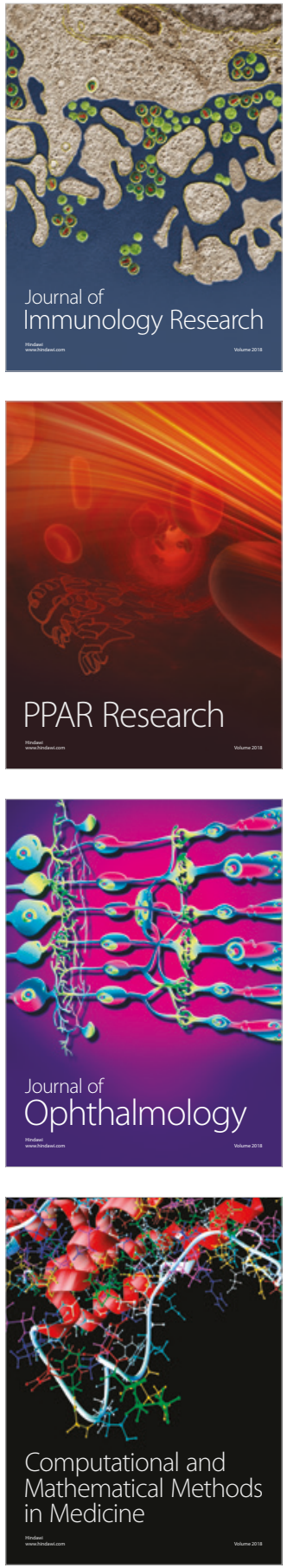

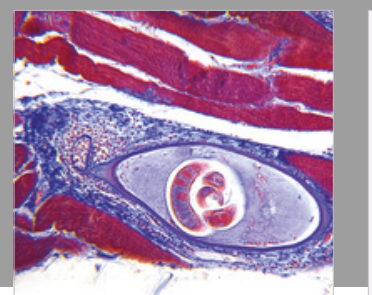

Gastroenterology Research and Practice

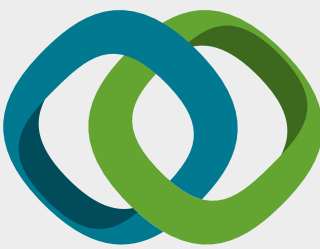

\section{Hindawi}

Submit your manuscripts at

www.hindawi.com
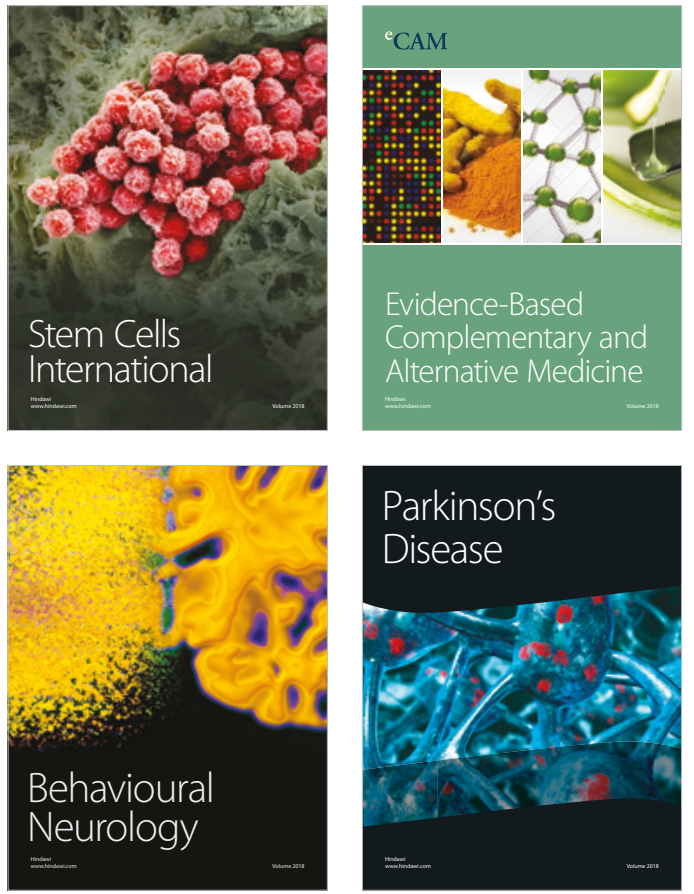

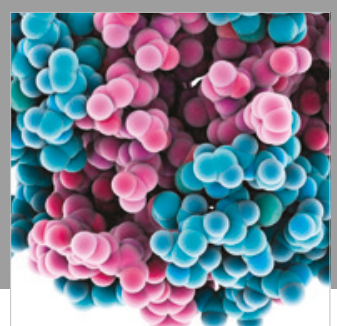

ournal of

Diabetes Research

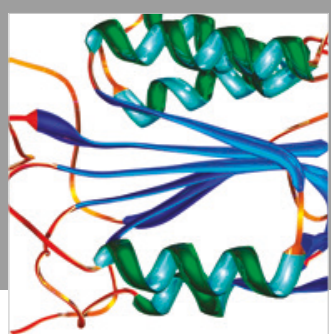

Disease Markers
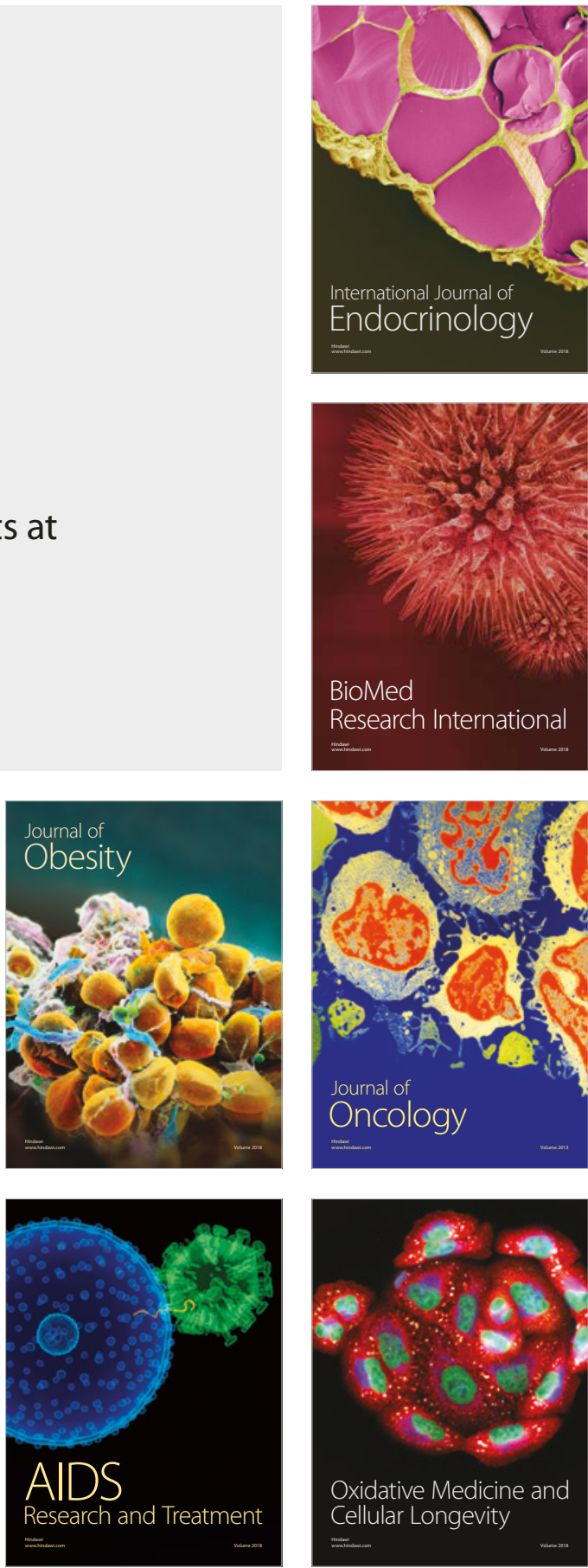